\title{
Fast Monotonic Blind Deconvolution Algorithm for Constrained TV Based Image Restoration ${ }^{\star}$
}

\author{
Haiying Liu ${ }^{\dagger}$, W.-S. Lu $u^{\ddagger}$, Yanan $\mathrm{Fu}^{\dagger}$, Yu Cheng ${ }^{\dagger}$, Tingfang $\mathrm{Yan}^{\dagger}$, Teng $\mathrm{Li}^{\dagger}$ and Max Q.-H. Meng ${ }^{\diamond \dagger *}$ \\ ${ }^{\dagger}$ School of Control Science and Engineering, Shandong University, Jinan, China \\ $\ddagger$ Dept. of Electrical and Computer Engineering, University of Victoria, Victoria, Canada \\ $\diamond$ Department of Electronical Engineering, Chinese University of Hong Kong, Hong Kong, P. R. China
}

\begin{abstract}
A new fast monotonic blind deconvolution algorithmic method is investigated based on the constrained variational minimization framework under the periodic boundary conditions. The contributions of our methodology are that the blur operator identification and image restoration can be simultaneously optimized even under high noise level as compared to previous methods. Specifically, the monotone fast iterative shrinkage/thresholding algorithm (MFISTA) combined with the fast gradient projection (FGP) algorithm, is extended to deal with our new proposed algorithm and guarantee the monotonic convergence rate. In addition, the deblurring subproblem is enhanced by incorporating a bisection technique to effectively identify a near optimal value for the regularization parameter of the TV-Frobenius objective function quickly and accurately. Initial experimental results for gray satellite and color wireless capsule endoscopy (WCE) images demonstrate the considerable performance of the proposed algorithm.
\end{abstract}

Index Terms-Blind Deconvolution, Total Variation, Deblurring, Denoising, Bisection Technique

\section{INTRODUCTION}

WCE is a sophisticated technique for diagnosing gastrointestinal tract diseases with practically no invasiveness [1]. It acquires images during a slow squirm process and transmits them by a wireless transmitter. However, raw WCE images are often contaminated by unknown kernel due primarily to the complicated situation of intestine and intrinsic restrictions of the equipment in terms of image acquisition and transmission. This in turn increases difficulties for accurate and effective diagnosis for the clinicians. Image blind deconvolution technique is an effective algorithmic procedure for unknown blur identification and image restoration.

Purpose of digital image restoration is to estimate the original image from the degraded observed image. The critical issue to solve this ill-posed inverse problem [2] is proper incorporation of prior knowledge about the original image into restoration process, that's why blind deconvolution still remains a complicated and challenging research topic in image processing. The generalized regularization approach using anisotropic diffusion has been proposed by You and Kaveh [3] based on $H^{1}$ norm and other TV regularization

${ }^{\star}$ This work is supported by the National High Technology Research and Development Program of China Grant \#2006AA040206. approaches [4] [5] are formulated with the aim of preserving edges by not over-penalizing discontinuities. After that, also based on TV minimization framework, Tony F. Chan and Chiu-Kwong Wong's algorithm [6] introduced the bounded variations blind deconvolution issues and treated image restoration problems. Lin He, Antonio Marquina and Stanley J. Osher [7] used TV regularization and Bregman iteration algorithm to solve blind deconvolution optimization problem. On the topic of image restoration using variational methods, we also refer to [8].

In this paper, we report some new developments for the restoration of images and identification of kernel based on variational minimization framework. First, based on periodic boundary conditions and TV minimization framework, the new blind deconvolution mathematical convex model is formulated in the specific inner product space, and the image and kernel are considered as two independent variables which can be optimized simultaneously. Second, the monotone fast iterative shrinkage/thresholding algorithm (MFISTA) combined with the fast gradient projection (FGP) algorithm, proposed recently in [9], is extended to deal with vector valued (color) images with several major changes and also is shown to exhibit fast theoretical and practical convergence rate. Third, once the optimal identified kernel is obtained, it is used for the general delurring subproblem to estimate the image incorporating a bisection technique [10] [11] to tune the regularization parameter of the TV-Frobenius objective function to its optimal value. Simulations are presented to demonstrate the performance of the new proposed algorithm.

The rest of the paper is organized as follows. Section II offers image model and previous related work. Section III describes the new proposed algorithm, the complexity algorithmic analysis and some necessary notations. Simulation results are demonstrated in comparison with existing blind deconvolution method in Section IV and Conclusions are given in Section $\mathrm{V}$.

\section{PRELIMINARIES}

\section{A. Image Model}

Consider a blurring model for discrete images

$$
\boldsymbol{f}=\boldsymbol{k} * \boldsymbol{u}+\boldsymbol{w}
$$


where $\boldsymbol{k}$ represents an affine map standing for a blurring operator, $\boldsymbol{f}$ denotes an observed noisy image, and $\boldsymbol{w}$ is normally distributed additive noise. The restoration problem here is to estimate (recover) image $\boldsymbol{u}$ given the observation $\boldsymbol{f}$, operator $\boldsymbol{k}$ and some statistical properties of noise $\boldsymbol{w}$ (such as mean and variance of $\boldsymbol{w}$ ).

It is well known [9] [12] [13] that the restoration at hand can be treated as an unconstrained convex optimization problem

$$
\underset{\boldsymbol{u}}{\operatorname{minimize}} \frac{1}{2}\|\boldsymbol{k} \boldsymbol{u}-\boldsymbol{f}\|_{F}^{2}+\mu\|\boldsymbol{u}\|_{\mathrm{TV}}
$$

where $\|\cdot\|_{F}$ denotes Frobenius norm of matrix, $\mu$ is regularization parameter that balances the trade-off between removing noise or small details, and $\|\boldsymbol{u}\|_{\mathrm{TV}}$ denotes the two-dimensional (2-D) discrete isotropic total variation of $\boldsymbol{u}$, which for a matrix $\boldsymbol{u}$ of size $n_{1}$ by $n_{2}$ is defined by

$$
\begin{aligned}
\|\boldsymbol{u}\|_{\mathrm{TV}}= & \sum_{i=1}^{n_{1}-1} \sum_{j=1}^{n_{2}-1} \sqrt{\left(u_{i, j}-u_{i+1, j}\right)^{2}+\left(u_{i, j}-u_{i, j+1}\right)^{2}} \\
& +\sum_{i=1}^{n_{1}-1}\left|u_{i, n_{2}}-u_{i+1, n_{2}}\right|+\sum_{j=1}^{n_{2}-1}\left|u_{n_{1}, j}-u_{n_{1}, j+1}\right|
\end{aligned}
$$

\section{B. Previous Work}

Restoration of image is usually a numerically ill-posed problem [2] [14] for general blur operator, blind deconvolution's main purpose is to estimate image and identify kernel without any priori information of original observed image.

There are many algorithms to solve the blind deconvolution for blur operator identification and image restoration. The last two decades have witnessed the growth of increasing research interest in this effective methodology and a large volume of literature covering a variety of image restoration problems. In 1996, Y.You and M.Kaveh [3] proposed the following model

$$
\min _{\boldsymbol{u}, \boldsymbol{k}} G(\boldsymbol{u}, \boldsymbol{k})=\frac{1}{2}\|\boldsymbol{k} \boldsymbol{u}-\boldsymbol{f}\|_{2}^{2}+\beta_{1}\|\boldsymbol{u}\|_{H^{1}}^{2}+\beta_{2}\|\boldsymbol{k}\|_{H^{1}}^{2}
$$

where $H^{1}$ norm is defined as

$$
\|\boldsymbol{u}\|_{H^{1}}^{2}=\int_{\Omega}|\nabla \boldsymbol{u}|^{2} d x d y=\int_{\Omega}\left(\boldsymbol{u}_{x}^{2}+\boldsymbol{u}_{y}^{2}\right) d x d y
$$

Because above functionals are continuous, variational calculus need to be solved by Partial Differential Equations (PDEs) for minimization which requiring heavy computation and this is practically a challenge research topic especially for tackling large scale image. The fatal weakness is that it can not yield a local minimizer. After that, You and Kaveh [3] and Tony F. Chan and Chiu-Kwong Wong [6] proposed the blind deconvolution algorithm using TV-minimization based techniques and this method is suitable for large-scale data. Originally in mathematical texts, TV is defined for functions of continuous variables that belong to a class known as functions of bounded variations (BV) [15], for convenience, we begin by reviewing the concept of their model,

$$
\min _{\boldsymbol{u}, \boldsymbol{k}} F(\boldsymbol{u}, \boldsymbol{k})=\frac{1}{2}\|\boldsymbol{k} \boldsymbol{u}-\boldsymbol{f}\|_{2}^{2}+\beta_{1}\|\boldsymbol{u}\|_{B V}^{2}+\beta_{2}\|\boldsymbol{k}\|_{B V}^{2}
$$

TV regularization works effectively for recovering "blocky" image [16] with consideration of keeping more critical sharp edges of the image. In [7], the authors developed alternating minimization algorithm to solve two EulerLagrange equations of $\boldsymbol{u}$ and $\boldsymbol{k}$ alternatively. During numerical experiments, they noticed the minimization problem may not have a unique solution. Simulations demonstrate a great improvement in quality of image and fast convergence of this algorithm after a few iterations. Practically, it still can not simultaneously identify the kernel and restored image or even worse the Bregman iteration will bring some noise back to the earlier round.

\section{Monotonic Gradient-Based Algorithms}

Of particular interest and relevance to the work reported here is a dual-based approach developed in [9] which yields a monotone fast iterative shrinkage-thresholding algorithm (MFISTA) where each iteration requires to solve a denoising subproblem which is carried out using a fast gradient projection (FGP) algorithm. The basic idea of [9] is to construct a dual problem in a way similar to that of A. Chambolle [17] where the unconstrained problem of minimizing the objective function without the box constraints was solved by a gradientbased algorithm. Due to the limitation in space, the reader is referred to paper [9] [18] for the algorithmic details of the MFISTA/FGP technique.

\section{NEW MODEL AND NUMERICAL ALGORITHM}

\section{A. Mathematical Problem Formulation}

In order to overcome shortcomings of nonsynchronous problems of the aforementioned methods, our new algorithm is proposed to address this issue by treating the image and kernel as two independent variables. The blind deconvolution problem is formulated as a boundary constrained convex problem based on the TV framework:

$$
\min _{\boldsymbol{u}, \boldsymbol{a}} F(\boldsymbol{u}, \boldsymbol{a})=\frac{1}{2}\|\boldsymbol{a} * \boldsymbol{u}-\boldsymbol{f}\|_{F}^{2}+\mu_{1}\|\boldsymbol{u}\|_{\mathrm{TV}}+\mu_{2}\|\boldsymbol{a}\|_{\mathrm{TV}}
$$

where $\boldsymbol{a}$ denotes the kernel and $\boldsymbol{u}$ is the original image, $\boldsymbol{u}_{0}$ is the noisy and blurred image, $*$ denotes the convolution operator, $\mu_{1}>0$ and $\mu_{2}>0$ are the regularization parameters for the TV norm of the image and kernel, respectively.

Suppose the image $\boldsymbol{u}_{k}$ and the kernel $\boldsymbol{a}_{k}$ is already known, $\boldsymbol{a}=\boldsymbol{a}_{k}+\triangle \boldsymbol{a}, \boldsymbol{u}=\boldsymbol{u}_{k}+\triangle \boldsymbol{u}, \triangle \boldsymbol{a}$ and $\triangle \boldsymbol{u}$ are small enough, then 


$$
\begin{aligned}
\boldsymbol{a} * \boldsymbol{u}= & \boldsymbol{a}_{k} * \boldsymbol{u}_{k}+\boldsymbol{a}_{k} * \triangle \boldsymbol{u}+\triangle \boldsymbol{a} * \boldsymbol{u}_{k}+\triangle \boldsymbol{a} * \triangle \boldsymbol{u} \\
= & \left(\boldsymbol{a}_{k} * \boldsymbol{u}_{k}+\triangle \boldsymbol{a} * \boldsymbol{u}_{k}\right)+\left(\boldsymbol{a}_{k} * \triangle \boldsymbol{u}+\boldsymbol{a}_{k} * \boldsymbol{u}_{k}\right) \\
& -\boldsymbol{a}_{k} * \boldsymbol{u}_{k}+\triangle \boldsymbol{a} * \triangle \boldsymbol{u} \\
= & \boldsymbol{a} * \boldsymbol{u}_{k}+\boldsymbol{a}_{k} * \boldsymbol{u}-\boldsymbol{a}_{k} * \boldsymbol{u}_{k}+\triangle \boldsymbol{a} * \triangle \boldsymbol{u} \\
\approx & \boldsymbol{a} * \boldsymbol{u}_{k}+\boldsymbol{a}_{k} * \boldsymbol{u}-\boldsymbol{a}_{k} * \boldsymbol{u}_{k}
\end{aligned}
$$

under above assumption, the quadratic part $\triangle \boldsymbol{a} * \Delta \boldsymbol{u}$ can be ignored temporarily for simple calculation purpose. Then problem (7) will be revised as

$\min _{\boldsymbol{u}, \boldsymbol{a}} F(\boldsymbol{u}, \boldsymbol{a})=\frac{1}{2}\left\|\boldsymbol{a} * \boldsymbol{u}_{k}+\boldsymbol{a}_{k} * \boldsymbol{u}-\boldsymbol{f}_{k}\right\|_{F}^{2}+\mu_{1}\|\boldsymbol{u}\|_{\mathrm{TV}}+\mu_{2}\|\boldsymbol{a}\|_{\mathrm{TV}}$

where $\boldsymbol{f}_{k}=\boldsymbol{a}_{k} * \boldsymbol{u}_{k}+\boldsymbol{f}$.

Assuming $\boldsymbol{x}=\{\boldsymbol{u}, \boldsymbol{a}\}, \mathcal{A}_{k} \boldsymbol{x}=\boldsymbol{a} * \boldsymbol{u}_{k}+\boldsymbol{a}_{k} * \boldsymbol{u}$ and $\|\boldsymbol{x}\|_{\mathrm{TV}}=\|\boldsymbol{u}\|_{\mathrm{TV}}+\|\boldsymbol{a}\|_{\mathrm{TV}}$, based on the inner product properties, then problem (9) can be rewritten by

$$
\min _{\boldsymbol{x}} F(\boldsymbol{x})=\frac{1}{2}\left\|\mathcal{A}_{k} \boldsymbol{x}-\boldsymbol{f}_{k}\right\|_{F}^{2}+\mu\|\boldsymbol{x}\|_{\mathrm{TV}}
$$

subject to :

$$
\begin{gathered}
\int \boldsymbol{a}(x) d x=1 \\
\boldsymbol{u}(x), \boldsymbol{a}(x) \geq 0 \\
\max \left(b_{l}, \boldsymbol{u}_{p, q}^{(k)}-\beta_{2}\right) \leq \boldsymbol{u}_{p, q} \leq \min \left(b_{u}, \boldsymbol{u}_{p, q}^{(k)}+\beta_{2}\right) \\
a_{i, j}^{(k)}-\beta_{1} \leq a_{i, j} \leq a_{i, j}^{(k)}+\beta_{1} \\
\boldsymbol{a}(x, y)=\boldsymbol{a}(-x,-y)
\end{gathered}
$$

where $\beta_{1}, \beta_{2}$ are two random small enough numbers and $b_{l}, b_{u}$ is the lower and upper boundary of the image separately. Then model (10) can be solved by two subproblems:

$$
\begin{aligned}
& \min _{\boldsymbol{u}} F(\boldsymbol{u})=\frac{1}{2}\left\|\mathcal{A}_{k} \boldsymbol{x}-\boldsymbol{f}_{k}\right\|_{F}^{2}+\mu_{1}\|\boldsymbol{u}\|_{\mathrm{TV}} \\
& \min _{\boldsymbol{a}} F(\boldsymbol{a})=\frac{1}{2}\left\|\boldsymbol{A}_{k} \boldsymbol{x}-\boldsymbol{f}_{k}\right\|_{F}^{2}+\mu_{2}\|\boldsymbol{a}\|_{\mathrm{TV}}
\end{aligned}
$$

\section{B. Algorithmic Details for Blind Deconvolution}

For the sake of predicting the feasibility and accuracy of our proposed blind deconvolution algorithm, this section is dedicated to the detailed mathematical derivation for our new methodology under the periodic boundary conditions [19]. As will become transparent shortly, the Gaussian blur matrix is essentially quadrantly symmetric version and this perfect property offers a significantly breakthrough for fast convergence rate of our algorithm. Hence, there are definitely some important revisions and totally different expressions of our new method particularly for the gradient part. According to Gaussian kernel's symmetric characters, the original kernel can be reformulated as the following size in order to reduce to the quantities the convolution operator parameters and this procedure will significantly accelerate the convergence rate of our algorithm.

Suppose the size of the original kernel $\boldsymbol{a}_{k}$ is $M \times M$, according to the symmetric characteristic of the Gaussian kernel matrix, the kernel size can be reduced dramatically from $M \times M$ to $M_{1} \times M_{1}$, where $M=2 m+1, M_{1}=m+1$, then the kernel can be denoted by $\widetilde{\boldsymbol{a}}_{k}$ with the size of $M_{1} \times M_{1}$

$$
\begin{aligned}
\widetilde{\boldsymbol{a}}_{k} & \triangleq\left[\begin{array}{c}
\boldsymbol{I}_{M_{1}} \\
\widehat{\boldsymbol{I}}
\end{array}\right] \boldsymbol{a}_{k}\left[\begin{array}{ll}
\boldsymbol{I}_{M_{1}} & \widehat{\boldsymbol{I}}^{\mathrm{T}}
\end{array}\right] \\
& =\widetilde{\boldsymbol{I}} \boldsymbol{a}_{k} \widetilde{\boldsymbol{I}}^{\mathrm{T}}
\end{aligned}
$$

where $\boldsymbol{I}_{M_{1}}$ is an identity matrix with the size of $M_{1} \times M_{1}$, $\widehat{\boldsymbol{I}}=\left[\begin{array}{ll}\widehat{\boldsymbol{I}}_{m} & \mathbf{0}\end{array}\right]_{m \times M_{1}}$ and $\widetilde{\boldsymbol{I}}=\left[\begin{array}{ll}\boldsymbol{I}_{M_{1}} & \widehat{\boldsymbol{I}}^{\mathrm{T}}\end{array}\right], \widehat{\boldsymbol{I}}_{m}$ can be obtained by flip an $m \times m$ identity matrix.

Based on the the above assumptions and definition of Frobieus norm, the fidelity term can also be described as

$$
\begin{aligned}
\left\|\mathcal{A} \widetilde{\boldsymbol{a}}_{k}-\boldsymbol{f}\right\|_{F}^{2}= & \operatorname{tr}\left(\widetilde{\boldsymbol{a}}_{k}^{\mathrm{T}} \mathcal{A}^{\mathrm{T}} \mathcal{A} \widetilde{\boldsymbol{a}}_{k}-2 \widetilde{\boldsymbol{a}}_{k}^{\mathrm{T}} \mathcal{A}^{\mathrm{T}} \boldsymbol{f}\right) \\
= & \operatorname{tr}\left(\boldsymbol{a}_{k}^{\mathrm{T}} \widetilde{\mathcal{A}}^{\mathrm{T}} \widetilde{\mathcal{A}} \boldsymbol{a}_{k}+\widehat{\boldsymbol{I}}_{k}^{\mathrm{T}} \widetilde{\mathcal{A}}^{\mathrm{T}} \widetilde{\mathcal{A}} \boldsymbol{a}_{k} \widehat{\boldsymbol{I}}^{\mathrm{T}}-2\left(\boldsymbol{a}_{k}^{\mathrm{T}} \widetilde{\mathcal{A}}^{\mathrm{T}} \widetilde{\boldsymbol{I}} \boldsymbol{f}\right)\right) \\
= & 2 \sum_{i=1}^{m}\left(\left(\boldsymbol{a}_{i}^{(k)}\right)^{\mathrm{T}} \widetilde{\mathcal{A}}^{\mathrm{T}} \widetilde{\mathcal{A}} \boldsymbol{a}_{i}^{(k)}\right)-2 \sum_{i=1}^{M_{1}}\left(\boldsymbol{a}_{i}^{(k)}\right)^{\mathrm{T}} q_{i} \\
& +\left(\boldsymbol{a}_{M_{1}}^{(k)}\right)^{\mathrm{T}} \widetilde{\mathcal{A}}^{\mathrm{T}} \widetilde{\mathcal{A}} \boldsymbol{a}_{M_{1}}^{(k)}
\end{aligned}
$$

where $q_{i}$ is the ith column of $q=\widetilde{\boldsymbol{I}}^{\mathrm{T}} \mathcal{A}^{\mathrm{T}} \widetilde{\boldsymbol{I}} \boldsymbol{f}$.

More specifically, the detailed mathematical derivations for the gradient expression of the above Frobieus norm can be expressed as

$$
\begin{aligned}
& \nabla_{\boldsymbol{a}_{k}}\left(\left\|\mathcal{A} \widetilde{\boldsymbol{a}}_{k}-\boldsymbol{f}\right\|_{F}^{2}\right)=2\left(2 \widetilde{\mathcal{A}}^{\mathrm{T}} \widetilde{\mathcal{A}}\right)\left(\widetilde{\boldsymbol{a}}_{k}\right)^{\mathrm{T}}-2 \mathcal{A}^{\mathrm{T}} \boldsymbol{f}\left[\begin{array}{c}
\boldsymbol{I}_{M_{1}} \\
\widehat{\boldsymbol{I}}
\end{array}\right] \\
& =2 \widetilde{\mathcal{A}}^{\mathrm{T}}\left(2 \widetilde{\mathcal{A}} \widetilde{\boldsymbol{a}}_{k}-\boldsymbol{f}\left[\begin{array}{c}
\boldsymbol{I}_{M_{1}} \\
\widehat{\boldsymbol{I}}
\end{array}\right]\right) \\
& =2 \widetilde{\mathcal{A}}^{\mathrm{T}}\left(\mathcal{A}\left(\widetilde{\boldsymbol{I}} \widetilde{\boldsymbol{a}}_{k} \widetilde{\boldsymbol{I}}-\boldsymbol{f} \widetilde{\boldsymbol{I}}\right)\right) \\
& =2 \widetilde{\boldsymbol{I}}^{\mathrm{T}} \mathcal{A}^{\mathrm{T}}\left(\mathcal{\mathcal { A }} \widetilde{\boldsymbol{a}}_{k}-\boldsymbol{f}\right) \widetilde{\boldsymbol{I}}
\end{aligned}
$$

where

$$
\widetilde{\boldsymbol{a}}_{k}=\left[\begin{array}{lllllll}
\boldsymbol{a}_{1}^{(k)} & \cdots & \boldsymbol{a}_{M_{1}-1}^{(k)} & \boldsymbol{a}_{M_{1}}^{(k)} & \boldsymbol{a}_{M_{1}-1}^{(k)} & \cdots & \boldsymbol{a}_{1}^{(k)}
\end{array}\right] .
$$

Actually, the mathematical derivation is more complicated for the above gradient expression (15) and the critical process is that the calculus results should be consistent with the original size of the kernel. Considering of the inner product property, we have

$$
\langle\mathcal{A} \boldsymbol{x}, \boldsymbol{v}\rangle=\left\langle\boldsymbol{x}, \mathcal{A}^{\mathrm{T}} \boldsymbol{v}\right\rangle
$$


where $\mathcal{A} \boldsymbol{x}=\boldsymbol{a} * \boldsymbol{u}_{k}+\boldsymbol{a}_{k} * \boldsymbol{u}$, then (16) can be reformulated as what follows

$$
\begin{aligned}
\left\langle\boldsymbol{a} * \boldsymbol{u}_{k}+\boldsymbol{a}_{k} * \boldsymbol{u}, \boldsymbol{v}\right\rangle & =\left\langle\boldsymbol{a} * \boldsymbol{u}_{k}, \boldsymbol{v}\right\rangle+\left\langle\boldsymbol{a}_{k} * \boldsymbol{u}, \boldsymbol{v}\right\rangle \\
& =\left\langle\boldsymbol{a}, \hat{\boldsymbol{u}}_{k} * \boldsymbol{v}\right\rangle+\left\langle\boldsymbol{u}, \hat{\boldsymbol{a}}_{k} * \boldsymbol{v}\right\rangle \\
& =\left\langle\{\boldsymbol{u}, \boldsymbol{a}\},\left\{\hat{\boldsymbol{a}}_{k} * \boldsymbol{v}, \hat{\boldsymbol{u}}_{k} * \boldsymbol{v}\right\}\right\rangle
\end{aligned}
$$

Due to the limitation of the paper format, formation (17) is discussed by two related short forms and please refer to the appendices (a) and (b) for the specific derivation processes.

\section{A Bisection Technique for Determining An Optimal Regularization Parameter}

Needless to say, using a good or, whenever possible, optimal value of regularization parameter $\mu$ is crucial as it affects the deblurring performance directly and significantly. The bisection technique described below is designed to determine a near optimal value of $\mu$ quickly for the deblurring subproblem using the obtained optimal kernel. The technique is based on the fact that $\mu$ is related to the variance of noise $w$ in a simple manner. For the sake of simplicity, we use model (1) and formulation (2) to illustrate the technique. It follows from (1) that if a solution $\boldsymbol{u}$ from a deblurring algorithm is in perfect agreement with the original noise-free and nonblurred image, it should satisfy

$$
\|\mathcal{A} \boldsymbol{u}-\boldsymbol{f}\|_{F}^{2}=\|\boldsymbol{w}\|_{F}^{2} \approx n_{1} n_{2} \sigma^{2}
$$

From (2), we see that parameter $\mu$ controls the trade-off between the TV-norm of the image and the closeness of $\boldsymbol{k} \boldsymbol{u}$ to $f$ in Frobenius norm: if $\mu$ is set to be too large, then (2) would put a heavier weight on the TV-norm term and, as a result, the second term $\frac{1}{2}\|\mathcal{A} \boldsymbol{u}-\boldsymbol{f}\|_{F}^{2}$ (known as fidelity term) gets too large, exceeding $\frac{1}{2} n_{1} n_{2} \sigma^{2}$ and violating (18); if $\mu$ is too small, then (2) would weigh the second term too heavy, leading to a $\|\mathcal{A} \boldsymbol{u}-\boldsymbol{f}\|_{F}^{2}$ considerably smaller than $n_{1} n_{2} \sigma^{2}$ that violates (18) again. Consequently, $\|\boldsymbol{A} \boldsymbol{u}-\boldsymbol{f}\|_{F}^{2}$ as a function of $\mu$ is a monotonic function that increases with $\mu$, and a near optimal value of $\mu$ can be identified as one that approximately satisfies (18). Based on the above analysis, a bisection technique for efficiently identify a near optimal value of $\mu$ is developed for the subproblem for deblurring issue, for the detailed information about bisection algorithm please refer to [10] [11].

Due to the consideration of the above analysis, a new fast proposed blind deconvolution technique for efficiency restoration of image and identification of blur operator can be readily sketched as follows:

Input : $\boldsymbol{f}, \delta$ function, $\mu_{1}, \mu_{2}, \beta_{1}, \beta_{2}$ and the tolerance $\varepsilon$.

Step 1: Iterate on $\mathrm{k}$ until convergence.

1) Solve problems (11)(12), get $\boldsymbol{u}_{k}$ and $\boldsymbol{a}_{k}$, respectively.
2) If satisfy the terminate condition, then stop; otherwise set $k=k+1$ and repeat from step 1 until obtain the approximate optimal kernel $\boldsymbol{a}_{\text {opt }}$.

Step 2: Proceed to the modified MFISTA/FGP algorithm once $\boldsymbol{a}_{\text {opt }}$ is obtained, then solve the iterative deblurring subproblem by incorporating the bisection technique until get the final estimated image $\boldsymbol{u}_{f}$.

\section{Remarks:}

(1) During implementation, the kernel must be proceeded to do symmetric step during every iteration.

(2) This algorithm possesses an exponential convergence rate of $1 / 2^{k}$.

\section{Simulations}

In this section, some numerical simulation results illustrate the efficiency and effectiveness of our new proposed fast blind deconvolution algorithm. Because the kernel identification is an ill-conditioned problem in the presence of observation noise, especially the high level noise which will make the situation even worse. This new technique demonstrates perfect simulation results and offers significant Signalto-Noise (SNR) improvement even the image is contaminated by the high level noise in comparison with methods [6].

A gray cameraman image of size $256 \times 256$ suffering a $9 \times 9$ Gaussian blur plus a small amount of additive Gaussian white noise with $\sigma=10^{-4}$ is shown in Fig.1(b). The algorithm was implemented in MATLAB. A Windows XP laptop PC with an Intel Core Duo CPU P8700@2.53 GHz with 2.0 GB of RAM, equipped with MALAB 7.8.0, was used to run the code. The differences $\beta_{i m a}=0.06, \beta_{k e r}=0.002$ are for the image and the kernel. The regularization parameters are $\mu_{i m a}=3 \times 10^{-4}$ and $\mu_{k e r}=1 \times 10^{-4}$, respectively. The nearly optimal kernel is acquired with the error difference of 0.0034 , then using the MFISTA/FGP method to solving the general deblurring issue. The restored image is shown in Fig.1(d). The SNR of the blurred and noise-corrupted image was $25.8101 \mathrm{~dB}$. We see that the SNR of the deblurred image was $37.4921 \mathrm{~dB}$ that offers $11.6820 \mathrm{~dB}$ improvement using the new algorithm based on MFISTA/FGP algorithm. The deblurred image performed by algorithm [6] is shown in Fig.1(c) with the improved SNR 30.6319 dB. The original kernel and identified one during the iterations are depicted in Fig.2(a) and Fig.2(b). The profiles of error difference and the objective functional value are exhibited in the Fig.3(a) and Fig.3(b). Observing the profile of the objective functional value, the monotonic convergence property will be obviously shown.

A color WCE image of size $140 \times 122$ suffering a $11 \times 11$ Gaussian blur plus a small amount of additive Gaussian white noise with $\sigma=10^{-4}$ is shown in Fig.4(b). Differences of the images and kernels, the regularization parameters of the TVFrobieus objective functional for the three different channels 
(a) Original image

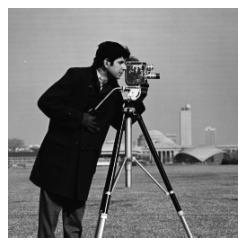

(c) Chan and Wong

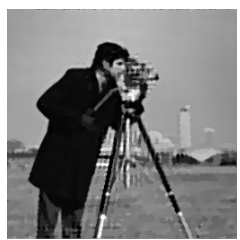

(b) Blurred image

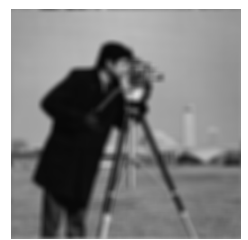

(d) Deblurred image

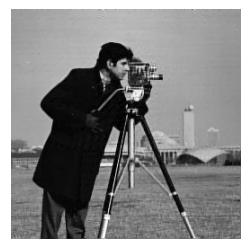

Fig. 1: Performance of the noise level of $\sigma=10^{-4}$.
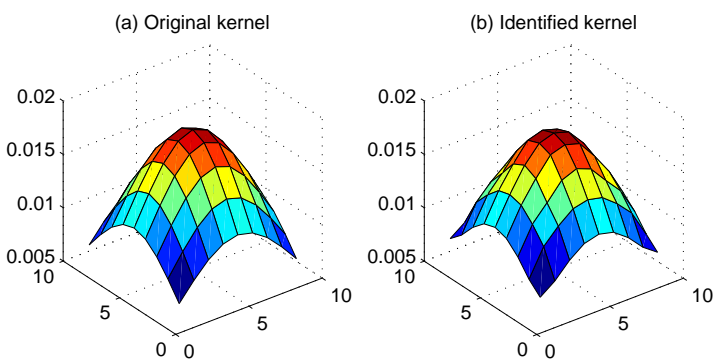

Fig. 2: Original and Identified kernel.
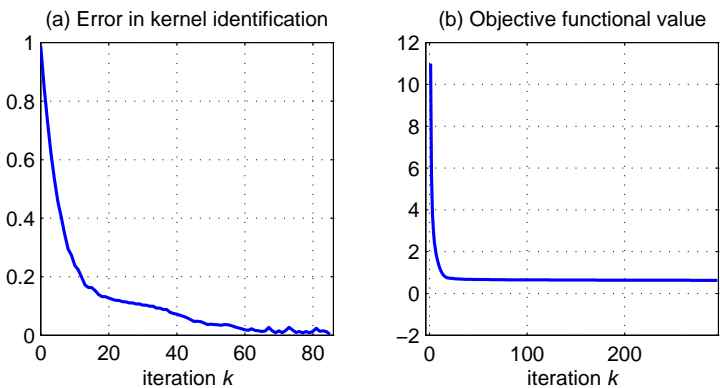

Fig. 3: Profiles of the objective functional value and the kernel error during the iterations.

in the RGB space are: red channel: $\left(\beta_{i m a}^{r}=0.04, \beta_{k e r}^{r}=\right.$ $\left.0.0025, \mu_{i m a}^{r}=2 \times 10^{-4}, \mu_{k e r}^{r}=4 \times 10^{-4}\right)$, green channel: $\left(\beta_{i m a}^{g}=0.01, \beta_{\text {ker }}^{g}=0.0015, \mu_{i m a}^{g}=1 \times 10^{-4}, \mu_{\text {ker }}^{g}=4 \times\right.$ $\left.10^{-4}\right)$, blue channel: $\left(\beta_{i m a}^{b}=0.005, \beta_{\text {ker }}^{b}=0.002, \mu_{i m a}^{b}=\right.$ $\left.2 \times 10^{-4}, \mu_{\text {ker }}^{b}=4 \times 10^{-4}\right)$. The restored image is shown in Fig.4(d). The SNR of the blurred and noise-corrupted image was $24.4758 \mathrm{~dB}$. We see that SNR of the deblurred image was $33.7907 \mathrm{~dB}$ that offered $9.3148 \mathrm{~dB}$ improvement. The SNR achieved by algorithm [6] was found to be $30.3642 \mathrm{~dB}$, a gain that was $3.4265 \mathrm{~dB}$ less than our new algorithm, the deblurred image with algorithm [6] is shown in Fig.4(c). The original kernel and the identified one during the iterations are depicted in Fig.5(a) and Fig.5(b). (a) Original image

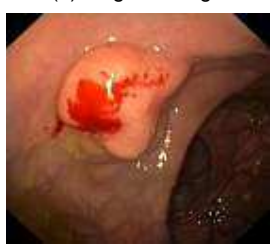

(c) Chan and Wong

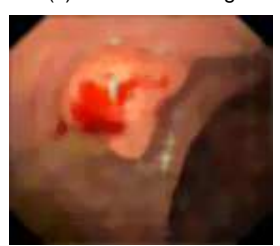

(b) Blurred noisy image

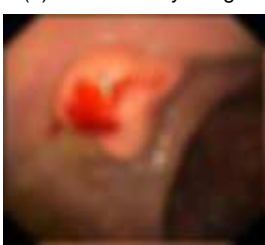

(d) Delurred image

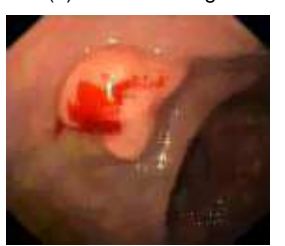

Fig. 4: Performance of the noise level of $\sigma=10^{-4}$.

The profiles of identified kernel errors for three channels during the iterations are depicted in Fig.5.

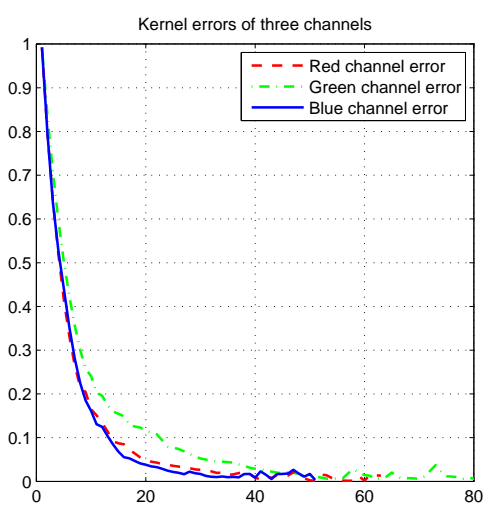

Fig. 5: The kernel errors for the three different channels

\section{CONCLUSIONS}

A new monotonic algorithm for blind deconvolution is proposed which possesses the capability of simultaneously kernel identification and image restoration under the periodic boundary condition. This method is built on a concept of color total variation in a MFISTA/FGP algorithmic 
framework, which guarantees the objective functional value possessing the monotonic property and fast convergence rate. Furthermore, once the optimal kernel is obtained, the bisection technique which is used for solving the subproblem of image deblurring issue and dedicating to determine an optimum regularization parameter rapidly and precisely. Several simulation studies in comparison with algorithm [6] are presented to evaluate the considerable performance gain of the proposed algorithm.

\section{ACKNOWLEDGMENT}

The authors would like to thanks a lot for the Dr. Jun Niu of Qilu Hospital of Shan dong University who offered plenty of medical professional instructions and affirmation for the blur operator identification and WCE image restoration.

\section{APPENDICES}

(a). For the specific derivation of the $\left\langle\boldsymbol{a}_{k} * \boldsymbol{u}, \boldsymbol{v}\right\rangle$, it is cast as

$$
\begin{aligned}
\left\langle\boldsymbol{a}_{k} * \boldsymbol{u}, \boldsymbol{v}\right\rangle=\sum_{p=1}^{n_{1}} \sum_{q=1}^{n_{2}}\left(\sum_{i=-m}^{m} \sum_{j=-m}^{m} \boldsymbol{a}_{i, j}^{(k)} \boldsymbol{u}_{p-i, q-j}\right) \cdot \boldsymbol{v}_{p, q} \\
=\sum_{i=-m}^{m} \sum_{j=-m}^{m} \boldsymbol{a}_{i, j}^{(k)}\left(\sum_{p^{\prime}=1-i}^{n_{1}-i} \sum_{q^{\prime}=1-j}^{n_{2}-j} \boldsymbol{u}_{p^{\prime}, q^{\prime}} \cdot \boldsymbol{v}_{p^{\prime}+i, q^{\prime}+j}\right) \\
=\sum_{i^{\prime}=m}^{-m} \sum_{j^{\prime}=m}^{-m} \boldsymbol{a}_{-i^{\prime},-j^{\prime}}^{(k)}\left(\sum_{p^{\prime}=1+i^{\prime}}^{n_{1}+i^{\prime}} \sum_{q^{\prime}=1+j^{\prime}}^{n_{2}+j^{\prime}} \boldsymbol{u}_{p^{\prime}, q^{\prime}} \cdot \boldsymbol{v}_{p^{\prime}-i^{\prime}, q^{\prime}-j^{\prime}}\right) \\
=\sum_{i=-m}^{m} \sum_{j=-m}^{m} \hat{\boldsymbol{a}}_{i, j}^{(k)}\left(\sum_{p=1+i}^{n_{1}+i} \sum_{q=1+j}^{n_{2}+j} \boldsymbol{u}_{p, q} \cdot \boldsymbol{v}_{p-i, q-j}\right) \\
=\sum_{i=-m}^{m} \sum_{j=-m}^{m} \hat{\boldsymbol{a}}_{i, j}^{(k)}\left(\sum_{p=1}^{n_{1}} \sum_{q=1}^{n_{2}} \boldsymbol{u}_{p, q} \cdot \boldsymbol{v}_{p-i, q-j}\right) \\
=\sum_{p=1}^{n_{1}} \sum_{q=1}^{n_{2}} \boldsymbol{u}_{p, q}\left(\sum_{i=-m}^{m} \sum_{j=-m}^{m} \hat{\boldsymbol{a}}_{i, j}^{(k)} \cdot \boldsymbol{v}_{p-i, q-j}\right) \\
=\left\langle\boldsymbol{u}, \hat{\boldsymbol{a}}_{k} * \boldsymbol{v}\right\rangle
\end{aligned}
$$

(b). For the detailed information of $\left\langle\boldsymbol{a} * \boldsymbol{u}_{k}, \boldsymbol{v}\right\rangle$, the detailed deduction is presented as

$$
\begin{aligned}
\left\langle\boldsymbol{a} * \boldsymbol{u}_{k}, \boldsymbol{v}\right\rangle & =\sum_{p=1}^{n_{1}} \sum_{q=1}^{n_{2}}\left(\sum_{i=-m}^{m} \sum_{j=-m}^{m} \boldsymbol{a}_{i, j} \boldsymbol{u}_{p-i, q-j}^{(k)}\right) \cdot \boldsymbol{v}_{p, q} \\
& =\sum_{i=-m}^{m} \sum_{j=-m}^{m} \boldsymbol{a}_{i, j} \sum_{p=1}^{n_{1}} \sum_{q=1}^{n_{2}} \boldsymbol{u}_{p-i, q-j}^{(k)} \cdot \boldsymbol{v}_{p, q} \\
& =\sum_{i=-m}^{m} \sum_{j=-m}^{m} \boldsymbol{a}_{i, j} \cdot \boldsymbol{w}_{i, j} \\
& =\left\langle\boldsymbol{a}, \hat{\boldsymbol{u}}_{k} * \boldsymbol{v}\right\rangle
\end{aligned}
$$

where $\boldsymbol{w}_{i, j}=\sum_{p=1}^{n_{1}} \sum_{q=1}^{n_{2}} \boldsymbol{u}_{p-i, q-j}^{(k)} \cdot \boldsymbol{v}_{p, q}, \boldsymbol{v}=\boldsymbol{f}_{k}$.

\section{REFERENCES}

[1] G. Iddan, G. Meron, A. Glukhovsky, and P. Swain, "Wireless capsule endoscopy," Nature, vol. 405, p. 417, 2000.

[2] C. Vogel and M. Oman, "Fast, robust total variation-based reconstruction of noisy, blurred images," Image Processing, IEEE Transactions on, vol. 7, no. 6, pp. 813-824, 1998.

[3] Y. You and M. Kaveh, "Anisotropic blind image restoration," in Image Processing, 1996. Proceedings., International Conference on, vol. 1. IEEE, 1996, pp. 461-464.

[4] _ - "A regularization approach to joint blur identification and image restoration," Image Processing, IEEE Transactions on, vol. 5, no. 3, pp. 416-428, 1996.

[5] — - "Blind image restoration by anisotropic regularization," Image Processing, IEEE Transactions on, vol. 8, no. 3, pp. 396-407, 1999.

[6] T. Chan and C. Wong, "Total variation blind deconvolution," Image Processing, IEEE Transactions on, vol. 7, no. 3, pp. 370-375, 1998.

[7] L. He, A. Marquina, and S. Osher, "Blind deconvolution using tv regularization and bregman iteration," International Journal of Imaging Systems and Technology, vol. 15, no. 1, pp. 74-83, 2005.

[8] T. Chan and C. Wong, "Convergence of the alternating minimization algorithm for blind deconvolution," Linear Algebra and its Applications, vol. 316, no. 1-3, pp. 259-285, 2000.

[9] A. Beck and M. Teboulle, "Fast gradient-based algorithms for constrained total variation image denoising and deblurring problems," Image Processing, IEEE Transactions on, vol. 18, no. 11, pp. 24192434, 2009.

[10] H. Liu, W. Lu, and M. Meng, "Fast algorithms for restoration of color wireless capsule endoscopy images," in Circuits and Systems (MWSCAS), 2011 IEEE 54th International Midwest Symposium on IEEE, 2011, pp. 1-4.

[11] — , "De-blurring wireless capsule endoscopy images by total variation minimization," in Communications, Computers and Signal Processing (PacRim), 2011 IEEE Pacific Rim Conference on. IEEE, 2011, pp. 102-106.

[12] L. I. Rudin, S. Osher, and E. Fatemi, "Nonlinear total variation based noise removal algorithms," Physica D: Nonlinear Phenomena, vol. 60, no. 1-4, pp. 259-268, 1992.

[13] L. Rudin and S. Osher, "Total variation based image restoration with free local constraints," in Image Processing, 1994. Proceedings. ICIP94., IEEE International Conference, vol. 1. IEEE, 1994, pp. 31-35.

[14] R. Chan, T. Chan, and C. Wong, "Cosine transform based preconditioners for total variation deblurring," Image Processing, IEEE Transactions on, vol. 8, no. 10, pp. 1472-1478, 1999.

[15] R. Acar and C. Vogel, "Analysis of bounded variation penalty methods for ill-posed problems," Inverse problems, vol. 10, p. 1217, 1994

[16] D. Dobson and F. Santosa, "Recovery of blocky images from noisy and blurred data," SIAM Journal on Applied Mathematics, pp. 1181-1198, 1996.

[17] A. Chambolle, "An algorithm for total variation minimization and applications," Journal of Mathematical imaging and vision, vol. 20, no. 1, pp. 89-97, 2004.

[18] A. Beck and M. Teboulle, "A fast iterative shrinkage-thresholding algorithm for linear inverse problems," SIAM Journal on Imaging Sciences, vol. 2, no. 1, pp. 183-202, 2009.

[19] M. Ng, R. Chan, and W. Tang, "A fast algorithm for deblurring models with neumann boundary conditions," SIAM Journal on Scientific Computing, vol. 21, no. 3, 1999. 